\title{
Enfermement des étrangers : l'Europe sous la menace du syndrome maltais
}

\section{Claire Rodier et Catherine Teule}

\section{(2) OpenEdition \\ 1 Journals}

\section{Édition électronique}

URL : http://journals.openedition.org/conflits/1752

DOI : 10.4000/conflits. 1752

ISSN : $1777-5345$

Éditeur :

CCLS - Centre d'études sur les conflits lilberté et sécurité, L'Harmattan

\section{Édition imprimée}

Date de publication : 1 mars 2005

Pagination : 119-155

ISBN : 2-7475-8103-9

ISSN : 1157-996X

\section{Référence électronique}

Claire Rodier et Catherine Teule, «Enfermement des étrangers : I'Europe sous la menace du syndrome maltais », Cultures \& Conflits [En ligne], 57 | printemps 2005, mis en ligne le 07 janvier 2010, consulté le 30 mars 2021. URL : http://journals.openedition.org/conflits/1752 ; DOI : https://doi.org/10.4000/ conflits. 1752

Ce document a été généré automatiquement le 30 mars 2021.

Creative Commons License 


\title{
Enfermement des étrangers : l'Europe sous la menace du syndrome maltais
}

\author{
Claire Rodier et Catherine Teule
}

1 Engagée depuis 1999, avec le traité d'Amsterdam, dans la mise en œuvre d'une politique commune d'asile et d'immigration, l'Union européenne (UE) était censée en boucler la première phase cinq ans plus tard*. Sur le plan formel, le programme prévu était en effet quasiment réalisé à la fin de l'année 2004: une dizaine de directives et de règlements communautaires ont été adoptés, concernant notamment l'accueil des demandeurs d'asile et les procédures qui leur sont applicables, les conditions d'accès au séjour des étrangers et des membres de leur famille, et le contrôle des frontières. Ces instruments sont loin de répondre aux objectifs exprimés par les chefs d'Etat et de gouvernement lors du sommet européen de Tampere, en octobre 1999. En quelques années, l'Union s'est en effet orientée vers une conception sécuritaire des frontières, enfermant les demandeurs d'asile dans la logique globalisante et restrictive de gestion des flux migratoires: "Les travaux [menés] par les Etats membres de l'Union européenne pour rapprocher leurs politiques d'asile - dont les organisations non gouvernementales comme les parlements nationaux sont souvent largement tenus à l'écart - sont déterminés par le contrôle des flux migratoires. Destinés à définir des normes communes à l'horizon 2004, ils ont prioritairement porté sur des mesures propres à entraver l'accès des demandeurs aux procédures d'asile, à éviter d'avoir à examiner leur demande et à mettre en place des formules de protection au rabais $»^{1}$.

2 Le $1^{\text {er }}$ mai 2004, dix nouveaux pays sont venus rejoindre l'Union européenne et forment ainsi, notamment à l'est avec les Etats baltes de l'ancien «bloc de l'est », la Pologne, la Slovaquie, la Hongrie, mais aussi au sud avec Malte et Chypre, une grande partie de sa nouvelle frontière avec les pays-tiers. Ceci va sans doute renforcer la tendance. Car l'élargissement, combiné avec les nouvelles règles com-munautaires en matière de détermination du pays responsable du traitement des demandes d'asile, risque d'avoir pour conséquence une répression accrue des migrants. Certains des nouveaux pays 
membres de l'UE sont en effet, de par leur histoire et leur situation économique, peu ouverts aux étrangers. Ce qui, en soi, ne constituerait pas forcément un problème s'ils n'étaient appelés, en application d'un mécanisme organisé par deux textes communautaires, le règlement dit «Dublin II $»^{2}$ et le règlement Eurodac ${ }^{3}$, à retenir ou à réadmettre sur leur sol la grande majorité des candidats à l'entrée dans l'UE qui auront transité par leur sol. Jusqu'à peu terres de passage des migrants vers l'Europe, et euxmêmes pays d'émigration, ils sont maintenant zones d'accueil et de traitement des demandes d'asile. Les efforts qu'ils ont été amenés à réaliser pour s'inscrire dans les normes communautaires de contrôle et de gestion des frontières n'y peuvent rien changer : le nombre de demandes d'asile décroît fortement dans l'UE à quinze, mais il croît fortement chez les dix nouveaux, notamment à Chypre et à Malte, en Pologne, en Slovénie et en République Tchèque ${ }^{4}$. Ainsi, alors que ces pays sont a priori moins bien équipés que les "anciens» Etats membres, tant sur le plan des traditions démocratiques que de l'infrastructure et de la logistique nécessaire à l'instruction des demandes d'asile, leur situation géographique les place en première ligne pour l'accueil des exilés, leur assignant en quelque sorte le rôle de cordon sanitaire de la vieille Europe. Le déséquilibre qui en résulte ne peut que laisser entrevoir des perspectives alarmantes si l'Union européenne, dans son ensemble, ne joue pas le jeu de la solidarité dans l'accueil des immigrants et des réfugiés.

3 On peut craindre, en effet, que devant faire face à des arrivées parfois massives d'étrangers tiers, les pays frontaliers tendent à s'en sortir par la pratique du refoulement déguisé, sous couvert par exemple de la légalisation commune des concepts de "pays sûr " ou d' « asile interne $»^{6}$. On observe déjà que l'idée de camps pour les étrangers se banalise. Admis tout d'abord comme inéluctables hors des frontières de l'Union, ils commencent à acquérir une certaine légitimité à l'intérieur. Certes, pour l'heure, le procédé est officiellement justifié par l'urgence ou le volume des populations à accueillir dans des pays mal préparés à les recevoir. Mais que les institutions européennes acceptent tacitement de telles solutions donne à penser que l'enfermement des étrangers immigrants, même lorsqu'ils demandent l'asile, pourrait devenir une norme. De là à considérer l'étranger qui veut rejoindre l'UE comme un criminel, le pas est étroit.

C'est en ce sens que les méthodes utilisées par la République de Malte pour assurer un contrôle des flux migratoires, qui reposent pour une bonne part sur la détention des migrants, et le fait qu'elles ne rencontrent aucune contestation officielle réelle, si ce n'est de forme, paraissent extrêmement inquiétantes. Il est à redouter que Malte, parce qu'elle concentre nombre des difficultés que commencent à rencontrer les nouveaux pays membres, ne préfigure des solutions à venir pour l'accueil des étrangers aux nouvelles frontières de l'Union, à savoir l'enfermement et le rejet. Pour prendre la mesure de cette menace, on se propose dans un premier temps d'examiner les conséquences désastreuses de la pratique de la détention administrative quasisystématique des migrants qui pénètrent illégalement sur le territoire maltais, puis dans un second temps de montrer comment cette pratique s'articule avec une politique d'asile destinée prioritairement à dissuader les étrangers de demander protection à Malte. En guise de conclusion, on présentera les recommandations formulées dans son rapport de mission par la FIDH, dont les observations sont à la base des développements qui suivent ${ }^{7}$. 


\section{La détention, instrument du contrôle migratoire}

5 Malte fait partie des dix pays de la dernière grande vague d'adhésion à l'Union européenne. Ile de la Méditerranée, c'est le plus petit de ces nouveaux entrants (plus petit que la britannique Ile de Wight); c'est aussi celui qui a la plus forte densité de population (395000 habitants, pour une surface de $\left.316 \mathrm{Km}^{2}\right)^{8}$. De par sa position géographique, c'est en outre une voie de passage naturelle des migrations du sud (Afrique, Moyen-Orient) vers l'Europe : située à $290 \mathrm{Km}$ de l'est de la Tunisie ou du nord de la Libye, elle n'est séparée du sud de la Sicile, et donc de l'Italie, que par $93 \mathrm{Km}$.

6 Ces données expliquent que ce pays connaît, depuis la fin des années 1980, des vagues d'arrivées d'étrangers, parfois importantes. Mais, pour la grande majorité de ces migrants, Malte n'est pas le but de leur voyage: ils y arrivent soit par hasard (chavirement des embarcations en Méditerranée ; opérations de récupération en pleine mer par l'armée ${ }^{9}$; passeurs les ayant lâchés sur les côtes), soit volontairement mais pour y trouver un moyen de rejoindre les côtes du continent européen.

7 Dans ce contexte, les autorités maltaises se disent matériellement incapables tant d'accueillir les étrangers qui débarquent par vagues successives que d'assurer leur éventuelle intégration sur le territoire. Ne disposant pas des structures ad hoc nécessaires, elles règlent la question par des mesures systématiques de placement en détention administrative des étrangers dits "illégaux ", à savoir la grande majorité des migrants qui arrivent dans ce pays, même lorsqu'ils sont demandeurs d'asile. Tout est fait pour qu'ils en repartent, volontairement ou au gré de mesures d'expulsion, et les conditions de détention qui leur sont appliquées reflètent cette volonté, même si leur précarité est officiellement justifiée par la nécessité de faire face à l'urgence et par l'absence de moyens.

\section{Les justifications de la pratique de la détention}

8 Le placement en détention des migrants est légalement admis. En effet, en vertu de l'Immigration Act, loi de 1970 modifiée en 2002, toute personne se trouvant sans titre sur le territoire (droit d'entrée, visa, titre de circulation, titre de séjour) est un immigrant «irrégulier» (prohibited immigrant). A ce titre, elle peut être placée en détention administrative en vue de son expulsion.

Immigration Act. Pour restreindre, contrôler, et réguler l'immigration à Malte et prévoir les questions qui y sont attachées.

« Toute personne, autre que celles ayant le droit d'entrer, ou le droit d'entrée et de séjour, ou de circulation et de transit (...) peut se voir refuser l'entrée, et (...) sera considérée comme un immigrant irrégulier » [section 5(1)]

"Lorsque le droit d'entrée (leave to land) est refusé à une personne arrivant à Malte par avion, cette personne sera placée temporairement en détention dans un lieu spécifique sur la terre (...) » [section 10(1)]

« Lorsque le droit d'entrée (leave to land) est refusé à une personne arrivant à Malte par tout autre moyen, cette personne peut à sa propre demande (...) être placée temporairement dans le pays et détenue dans un lieu spécifique (...) » [section 10(2)] «Toute personne détenue en vertu des paragraphes (1) ou (2) sera considérée comme étant en détention administrative et non comme ayant atterri ». [section 10(3)]

«Si une personne est considérée par l'officier Principal d'Immigration (Principal Immigration Officer) comme étant sujette à l'éloignement, par son statut d'immigrant 
irrégulier, en vertu des provisions de l'article 5 (...), ledit officier peut émettre un ordre d'éloignement contre une telle personne (...)» [section 14(1)]

«Lorsque un tel ordre est donné, la personne à qui il est adressé sera en détention préventive avant de devoir quitter Malte ». [section 14(2)]

9 Comme on le verra, ce texte s'applique indifféremment aux immigrés dits "illégaux » et aux demandeurs d'asile, la loi sur l'asile adoptée en 2001 (Refugees Act ${ }^{10}$ ) ne prévoyant aucune clause de protection spécifique pour ces derniers, dès lors qu'ils sont entrés « illégalement » sur le territoire. Le gouvernement maltais s'en justifie par le fait que la quasi-totalité des étrangers arrivant à Malte demandent l'asile. Il estime donc qu'il n'y a pas de réelle distinction à établir entre deux types de population; ce qui revient à considérer que les demandeurs d'asile sont, a priori, des migrants illégaux.

10 Face aux critiques, il ajoute ne pas avoir d'autres solutions en raison de l'accroissement du nombre d'étrangers arrivant sur le territoire (" The country is not prepared for such an influx $»^{11}$ ). Mais l'argument de l'urgence et de la situation de crise perd quelque peu de sa force lorsque l'on sait que, depuis plusieurs années déjà, Malte enregistre des flux saisonniers d'arrivées de migrants, que le phénomène est donc bien connu des autorités, et que les situations dites de «crise » se répètent régulièrement...

11 Aussi, Tonio Borg, ministre de l'Intérieur, invoque-t-il également des raisons de sécurité. Il estime, en effet, qu'en laissant les migrants en liberté cela risquerait de "créer plus de problèmes, non seulement pour Malte, mais aussi pour les pays voisins ${ }^{12}$.Le même argument est repris dans la réponse de Malte au Commissaire des droits de l'Homme du Conseil de l'Europe : « il n'est pas dans l'intérêt de Malte de changer sa politique de détention. Si [les migrants] avaient été immédiatement relâchés, ils auraient causé des perturbations sur le marché du travail et des problèmes importants quant à leur hébergement $»^{13}$.

12 Il faut dire que l'analyse du gouvernement maltais paraît admise - parfois même partagée - par les différents observateurs internationaux qui ont réalisé des rapports sur Malte au cours des dernières années. C'est également l'avis de l'Ombudsman maltais. En effet, si les uns et les autres portent en général de sévères appréciations sur les conditions de détention imposées aux étrangers par Malte, ils n'en paraissent pas moins adhérer à l'idée que les spécificités de ce pays expliquent le dispositif d'exception mis en place à l'égard des migrants et des demandeurs d'asile. Au mieux, recommandent-ils au gouvernement de s'orienter vers la recherche de solutions alternatives à la détention administrative de ces derniers. Il en est de même pour les autorités européennes qui, amenées à se prononcer sur la situation maltaise dans le cadre des rapports d'évaluation de la pré-adhésion, paraissent, elles aussi, tenir le système de détention des étrangers « illégaux » pour un fait acquis sinon légitime, et se sont limitées à recommander de l'encadrer légalement ${ }^{14}$.

13 La détention est donc appliquée sans discrimination et l'on trouve dans les centres maltais des hommes, des femmes (parfois enceintes), des familles avec enfants, des mineurs isolés, comme des personnes appartenant pourtant aux groupes dits «vulnérables $»^{15}$, et répondant aux statuts les plus divers :

14 - étrangers sans titre lors de leur entrée sur le territoire et auxquels a été signifié un refus d'entrée ;

15 - étrangers interpellés sur le territoire, soit sans titre de séjour à la suite d'une entrée illégale, soit après expiration du délai de validité de leur autorisation de séjour ; 
16 - étrangers repêchés en mer par les autorités militaires à la suite de difficultés de navigation ou d'opérations d'arraisonnement ;

17 - étrangers ayant déposé une demande d'asile ou de protection humanitaire ou temporaire, après signification d'une interdiction d'entrée ou interpellation sur le territoire ;

18 - demandeurs d'asile déboutés par le Commissaire aux réfugiés et en attente du résultat de la procédure d'appel. C'est, de l'avis de l'ensemble des témoins, la catégorie numériquement la plus nombreuse, en raison notamment de la durée de la procédure d'appel ;

19 - demandeurs définitivement déboutés en appel, refusant de quitter le territoire et non expulsables ;

20 - étrangers sous arrêté d'expulsion, particulièrement ceux dont le renvoi est suspendu à la confirmation de leur identité par les autorités de leur pays d'origine ;

21 - étrangers « volontaires » au retour, mais qui ne peuvent régler le montant des frais de transport ;

22 - mais aussi demandeurs d'asile ayant obtenu une protection (conventionnelle ou humanitaire) et pour lesquels il n'y a pas de place d'hébergement disponible.

23 Cette conception de la dissuasion par l'enferment est d'autant plus inquiétante que l'adhésion de Malte à l'Union européenne risque moins d'apporter des solutions directes aux problèmes que pose l'afflux des étrangers que de les aggraver. En effet, en entrant dans l'UE et compte tenu de sa position géographique, Malte a renforcé son statut de terre de transit ; cette île est et restera un point de passage dans les parcours des migrants d'Afrique et du Moyen-Orient vers l'Europe, quand bien même des accords de réadmission seraient-ils conclus avec la Libye ou d'autres pays du pourtour méditerranéen ${ }^{16}$. Dans le même temps, Malte est entrée dans «l'espace Dublin ${{ }^{17}}^{17}$ et devra donc appliquer la règle du premier pays d'entrée dans l'UE, à savoir assurer l'instruction des demandes d'asile et d'immigration de tous ceux qui auraient " transité » par son territoire; et elle devra offrir aux réfugiés reconnus les moyens de vivre sur son territoire. Quant aux étrangers qui, après être passés par Malte, auraient trouvé le moyen de se «dissoudre » dans la masse des migrants qui circule en Europe, ils devraient être refoulés du pays de l'UE où ils se trouveront pour être « remis » aux autorités maltaises ; à charge pour celles-ci de procéder à leur éloignement. Dans l'un et l'autre cas, Malte se trouvera donc contrainte de gérer un flux d'immigrants dont rien ne permet d'envisager la décroissance et dont elle estime qu'elle n'a pas les moyens de le prendre en charge.

\section{Une solution dite d'urgence, mais pérenne}

24 Si les bases juridiques de cette politique de privation de liberté des étrangers ne suscitent pas beaucoup de contestations internationales et aucun débat politique national, en revanche, les conditions de vie dans les centres de détention sont très largement et sévèrement critiquées tant elles se révèlent inacceptables au regard du respect de la dignité humaine. Depuis quelques années, les rapports se multiplient pour les dénoncer: Ombudsman maltais, Conseil de l'Europe, $\mathrm{CPT}^{18}$, Amnesty International. Et c'est d'abord sur cette question que la FIDH a mandaté une mission en février 2004, 
avant de constater que le problème dépassait le cadre de la détention pour couvrir l'ensemble du champ de la politique migratoire et particulièrement de l'asile.

A l'origine, le système était conçu pour une centaine de personnes et son administration relevait en principe de la police. La plupart des immigrants "illégaux » étaient donc regroupés à Ta'Kandja, QG de la police. Mais lors d'une visite effectuée en mai 2001, le CPT a préconisé la fermeture de ce centre, jugeant ses conditions matérielles inacceptables ( locaux (...) caractérisés par la saleté et le délabrement (...) situation misérable $»)^{19}$. Malte a donc spécialement aménagé en 2002 la caserne de Hal Far, dans l'objectif déclaré d'y accueillir l'ensemble des demandeurs d'asile et immigrants irréguliers. Mais ce centre est notoirement insuffisant puisqu'il n'offre qu'une capacité de 60 à 80 places. En conséquence, les solutions "provisoires" se multiplient par réquisition de bâtiments, le plus souvent dans des enceintes militaires, inadaptés pour accueillir des détenus de longue durée, et le centre de Ta'Kandja a connu quelques travaux et n'a pas été fermé comme le demandait le CPT.

La dénomination de "camp " que nous utiliserons ici, en référence aux travaux menés par les chercheurs appartenant au réseau Migreurop ${ }^{20}$, recouvre donc une réalité matérielle assez diverse : des casernes, des baraquements, parfois même des tentes en période de forte affluence. La liste des personnes détenues dans ces camps pour immigrants est confidentielle et aucune statistique officielle ne permet d'avoir une mesure exacte de l'ensemble du phénomène. Les chiffres fluctuent très vite et très amplement au gré des renvois groupés ou des régularisations, mais aussi des arrivées saisonnières ${ }^{21}$. Mais tous les témoignages concordent pour dire que les centres sont surpeuplés.

27 Les cinq camps identifiés en février 2004 :

28 - Hal Far : aménagé à partir de l'été 2001, il est véritablement entré en fonction à partir de février 2002. Y coexistent un centre de rétention sous autorité de l'armée qui n'accueille que des célibataires (officiellement 80 personnes au maximum, mais souvent beaucoup plus selon l'Ombudsman) et, juste à l'extérieur, un centre ouvert ;

29 - Hal Safi : caserne sous autorité militaire où sont placées quelques familles, en plus des célibataires ;

30 - Lyster Barracks : camp sous autorité militaire et composé, outre d'une caserne, d'un ensemble de baraquements. A l'origine, il avait été aménagé pour recevoir des familles - parfois avec femme enceinte - et des femmes seules. Les hommes célibataires n'y ont été placés que plus tardivement. Il a accueilli jusqu'à 230 demandeurs d'asile. Les conditions de détention dans ce centre ont notamment été dénoncées par le Jesuit Refugee Service (JRS). Il n'était pas utilisé en février 2004, lors de la mission FIDH.

31 - Floriana : au quartier général de la Police (300 à 400 personnes); la presse s'est largement fait l'écho des conditions de vie des détenus à la suite d'une tentative d'évasion. Il était également fermé à l'époque de la mission FIDH.

32 - Ta'Kandja (Siggiewi) : placé sous l'autorité de la police. Dans son rapport 2002, à l'instar du CPT, l'Ombudsman estimait également que ce centre était inadapté à l'accueil de détenus. En 2004, il semble pourtant toujours utilisé, les autorités maltaises ayant fait valoir dans leur réponse au CPT (juillet 2003), comme précédemment, devoir faire face à une "situation d'urgence" engendrée par les arrivées en nombre d'étrangers ( $A \mathrm{Au}$ vu de la situation inhabituelle et exceptionnelle rencontrée cette année (...) il n'y avait pas d'autre alternative à son utilisation (...). Il sera fermé dès que possible ${ }^{22}$. Ce 
centre figure à nouveau dans la liste des lieux inspectés par le CPT lors de sa visite de janvier 2004 et dont les conclusions ne sont pas encore publiques. définissant des normes de fonctionnement, et notamment les droits des prisonniers, ou les limites au pouvoir de décision des gardiens. Par exemple, dans les centres placés sous autorité militaire, un règlement intérieur (du 14 mars 2002) fixe l'emploi du temps des détenus (repas, nettoyage, récréations, repos) et les mesures à prendre en cas de maladie. Rien de tel dans les centres sous autorité policière, et les détenus ou les témoins extérieurs indiquent une différence notable des pratiques des gardiens selon qu'ils sont policiers ou militaires. Ces derniers imposent des règles strictes mais sont mieux préparés à assurer la protection des personnes, alors que les policiers paraissent plus souples dans leur organisation, mais sont aussi moins rigoureux. De même, l'organisation varie largement selon les camps, l'autorité de tutelle, ou le directeur, comme elle varie dans le temps en fonction de l'affluence. C'est dire que la gestion de chaque centre repose sur un certain arbitraire dans l'appréciation de la situation et selon celui qui l'apprécie.

37 Cette absence de règles communes - et donc de possibilité de contrôle - pourrait évidemment être une source de graves dysfonctionnements d'autant que les " gardiens » ne sont qu'occasionnels. Préparés pour d'autres métiers, ils ignorent tout du droit des étrangers, comme ils méconnaissent les conséquences psychologiques de cet enferment sur les détenus. Le fait que des actes de violence des gardiens à l'égard des détenus ne soient pratiquement jamais signalés et que, au contraire, de nombreux témoignages - y compris de détenus - indiquent que certains gardiens font manifestement leur possible pour rendre moins inhumaine la situation d'enfermement des étrangers, doit donc être souligné.

\section{Des conditions de détention dénoncées comme inhumaines et dégradantes}

38 Aucun de ces centres n'a été conçu pour la détention de centaines de personnes, célibataires des deux sexes, mineurs non-accompagnés, femmes enceintes et familles avec enfants, a fortiori pour de longues périodes. Même celui de Hal Far, pourtant « aménagé » en 2002, est sévèrement critiqué par A. Gil-Roblès, commissaire aux droits de l'Homme du Conseil de l'Europe : «Les conditions rencontrées dans le centre Hal Far (...) sont en grande partie similaires en matière de surpopulation et de promiscuité, d'absence d'activités mais aussi de salubrité. J'ai pu constater que les personnes détenues dans ce centre vivaient, elles aussi, de façon entassée et que le centre était dénué de structures adéquates à l'accueil et à la récréation d'un nombre si important de détenus. (...) On peut considérer que le constat que j'ai pu faire sur les mauvaises conditions de détention des migrants peut être étendu à l'ensemble des sites où sont retenus les migrants $»^{23}$. 

aucune possibilité d'isolement et de vie privée, au point que à Lyster Barracks et à Safi, où sont accueillies des familles, l'absence de cloisonnement est palliée par des draps suspendus entre les lits. Quand la place manque, des pièces de service (couloir, cuisine, réserve) sont réquisitionnées (Ta'Kandja, 2002). A Hal Safi, le centre a hébergé jusqu’à 320 personnes en novembre 2002, obligeant à loger les migrants sous des tentes pendant l'hiver ou dans des baraques en tôle qualifiées, de l'aveu même des autorités administratives, de réfrigérateur en hiver et de four en été.

Si à Hal Safi, A. Gil-Robles a pu observer une tentative de la direction du camp pour organiser la répartition des détenus par affinités nationales, linguistiques ou religieuses, dans d'autres camps l'espace est beaucoup trop restreint pour le faire. Au bruit général d'une foule dense vivant dans un espace confiné, où la télévision fonctionne sans arrêt (jour et nuit), s'ajoute donc la cacophonie linguistique et les altercations, certains détenus estimant que les ressortissants de quelques pays bénéficient d'un meilleur traitement qu'eux (par exemple, les Erythréens ${ }^{24}$ comparés aux Irakiens ou aux Soudanais). L'ambiance est en permanence survoltée et ne manque pas d'induire une tension nerveuse extrême chez les gardiens chargés d'assurer la sécurité des lieux.

41 Dans un tel contexte de surpopulation et de promiscuité, mais aussi par manque de moyens, les conditions d'hygiène laissent nécessairement à désirer et se révèlent largement inférieures aux standards internationaux. La fréquence d'accès aux douches, par exemple, est très limitée en raison de l'insuffisance d'équipements sanitaires. Idem pour l'état des toilettes, difficiles à maintenir propres dans un contexte d'utilisation intensive.

Ce sont les détenus qui doivent assurer le nettoyage des locaux. De même, doivent-ils procéder à la lessive de leur linge ; mais, lors de sa visite en avril 2002, l'Ombudsman ${ }^{25}$ a pu constater que soit il n'y avait pas de lave-linge à leur disposition (Lyster Barracks, Ta'Kandja), ce qui posait un problème évident aux familles avec enfants, soit la machine existante était en panne depuis le début de l'ouverture du camp (Safi). A Floriana, la lessive se fait dans la cour dans des cuvettes en plastique et le linge est mis à sécher sur les barbelés.

Ce sont, en fait, les seules activités offertes aux étrangers détenus. Ils passent donc leur temps à traîner et à se morfondre, dans le bruit de la télévision. D'autant que dans les camps militaires, et pour des raisons de sécurité, ils ne peuvent prendre l'air qu'à l'heure de la "récréation » autorisée. Mais même si, dans les centres gérés par la police, les chambres/dortoirs étant le plus souvent ouverts dans la journée, les migrants peuvent librement circuler dans l'enceinte du camp, les espaces de détente en plein air sont restreints et très insuffisants pour permettre une réelle activité physique à une population si importante. L'Ombudsman reconnaît que les cours sont très petites et ressemblent à celles des prisons. A Hal Far, caserne pourtant spécialement aménagée, les 80 détenus n'ont accès qu'à un demi terrain d'entraînement de basket encerclé de grillage et à une petite cour dans laquelle ils ne peuvent que tourner en rond. La même observation peut être appliquée à Safi. A Floriana, même chose : une toute petite cour grillagée, tellement surchauffée en été qu'il est impossible d'y rester. Seul Ta'Kandja paraît offrir de meilleures conditions, avec un terrain un peu plus grand.

Dans un tel contexte de promiscuité et de surpopulation, le risque d'épidémie et de contagion est extrême, font remarquer tous les observateurs. « Les personnes atteintes de 
maladies contagieuses et ponctuelles ne sont pas suffisamment séparées des autres migrants, ce qui rend le risque de propagation des maladies beaucoup plus important ${ }^{26}$. Or, il n'y a aucune antenne médicale ou infirmière sur place et la Croix-Rouge qui venait une ou deux fois par semaine dans certains camps a interrompu ses visites. L'accès aux soins constitue donc une véritable préoccupation pour les détenus. Et, dans ces conditions, gale, poux, vers, grippes constituent leur lot quotidien. Qui plus est, lorsque prescription médicale il y a, elle est administrée par les gardiens des camps ${ }^{27}$ : ils achètent les médicaments et les distribuent, sans qu'aucune formation ne les ait préparés à cette fonction d'auxiliaire médical et sans pouvoir contrôler que les médicaments sont pris au bon moment et régulièrement; or, nombre de migrants ne lisent ni le maltais ni l'anglais et ne peuvent donc comprendre les indications de posologie figurant sur les notices. Selon le Jesuit Refugee Service (JRS) ${ }^{28}$, certains détenus sont pourtant dans un état de santé incompatible avec un régime de détention où ils ne peuvent bénéficier d'aucun soin journalier, d'aucun régime alimentaire et d'aucun isolement. Or, pour des raisons de logistique et de sécurité, l'accès aux établissements hospitaliers est très difficile, même en cas de pathologies déclarées.

Pourtant, ce ne sont pas tant ces conditions matérielles indécentes qui suscitent des protestations de la part des étrangers que l'incertitude dans laquelle ils se trouvent sur la durée de leur détention, particulièrement pour les demandeurs d'asile. Les détenus attendent sans rien faire pendant de longs mois, ne sachant pas où en est leur dossier, ignorant tout de l'échéance à laquelle ils seront fixés sur leur sort.

Cette anxiété permanente trouve sa traduction la moins grave dans les différents maux d'origine psychosomatique dont se plaignent les détenus. Mais le JRS constate surtout une augmentation des prescriptions d'anxiolytiques et d'antidépresseurs et des demandes d'admission à l'hôpital psychiatrique de Mont Carmel. Nombre de détenus, y compris des enfants mineurs, manifestent des pulsions suicidaires avec le risque latent d'un effet de contagion sur les autres. Episodiquement, certains d'entre eux utilisent l'arme de la grève de la faim pour essayer de se faire entendre.

Une étude menée en Australie pendant 18 mois montre bien que dans les centres de détention, le taux de suicide est largement supérieur à celui de la population générale, notamment chez les jeunes, et que les problèmes de santé mentale y croissent de façon exponentielle avec la durée de la détention ${ }^{29}$. Ce phénomène, que certains appellent "syndrome de dépression collective ", peut conduire à des problèmes autrement plus graves que ceux qui sont actuellement observés dans les camps de Malte, non seulement pour les étrangers détenus mais aussi pour le personnel chargé de les surveiller.

\section{L'obsession de la sécurité}

Surpopulation, inactivité des détenus, attente sans terme fixé, induisent un climat de tension permanent. Tous les témoins admettent que les conditions de vie dans les centres nécessitent une vigilance constante de la part des gardiens qui redoutent les actes de violence et les tentatives d'évasion. D'autant que, dans ce système de détention organisé continûment en urgence, le nombre de gardiens affecté à chaque centre ne paraît pas évoluer proportionnellement au nombre d'arrivants.Le moindre incident est donc redouté par les gardes parce qu'il risque de déclencher des réactions de révolte des détenus dont témoigne une succession de tentatives d'évasion en juillet 2003 (Floriana, le 19 juillet : 54 détenus; Ta'Kandja, le 27 juillet : 23 personnes), faisant suite 
à celle de 27 Erythréens en novembre 2002. Tout événement peut prendre des proportions dramatiques. Il en a été ainsi, par exemple, lors de l'annonce de la libération des Erythréens en décembre 2003 : à la surprise a succédé, chez les Soudanais qui attendaient depuis plus longtemps - deux ans et demi - un sentiment de désespoir.

Dans ce contexte, il faut remarquer que les allégations de violences commises par des gardiens sont fort peu nombreuses. Il n'en reste pas moins que l'objectif de sécurité conduit les autorités administratives des camps à prendre des mesures qui ne peuvent qu'être jugées humiliantes par les détenus et qui ont été pointées tant par l'Ombudsman que par le Commissaire aux droits de l'Homme du Conseil de l'Europe : obliger les détenus à retirer les lacets de leurs chaussures dans certains centres (Ta'Kandja), mais surtout faire systématiquement usage des menottes pour toute sortie du camp, même pour les personnes malades qui doivent être conduites à l'hôpital. Certains étrangers refusent donc d'aller consulter les médecins pour ne pas être vus ainsi. A cette critique, les autorités maltaises se contentent de répondre que "des moyens seront trouvés pour rendre la pratique plus acceptable $»^{30}$.

En tout état de cause, les sorties du camp sont rares car elles nécessitent une organisation lourde : réquisitionner un véhicule, prévoir une escorte qui accompagne les détenus. C'est pour cette raison que les enfants mineurs qui, en principe, devraient être scolarisés, attendent souvent plusieurs mois avant de pouvoir accéder à un établissement. La difficulté est identique pour les détenus malades qui souhaitent aller en consultation à l'hôpital ou ont besoin de soins ; ils doivent réitérer leur demande, la faire appuyer par un avocat, avant d'obtenir satisfaction. Et, là aussi la sécurité prime : ils arrivent donc menottés à l'hôpital, et y séjournent dans des conditions qui ne sont guère différentes de l'incarcération, strictement surveillés dans leur chambre vingtquatre heures sur vingt-quatre par cinq policiers. Ces étrangers ne sont pourtant pas des criminels, mais comme le dit l'un des responsables du JRS, « on a aussi enraciné dans l'esprit des gens que ces personnes sont criminelles $»^{31}$, et les personnels soignants semblent ne pas échapper à cette crainte, d'autant qu'il est rare qu'ils puissent comprendre leur langue.

51 Théoriquement, les détenus peuvent recevoir des visites. Mais selon les camps, selon les périodes, l'administration accorde plus ou moins facilement les autorisations nécessaires. Parfois, lorsque les surveillants les y autorisent, c'est au travers des grillages des cours, à l'heure de la "récréation ", qu'ils parviennent à parler avec les personnes extérieures. Les ONG, elles-mêmes ont dû, à certaines époques, demander à l'Ombudsman d'intervenir auprès du ministre de l'Intérieur pour franchir la barrière des tracasseries administratives qui leur étaient opposées.

52 Pour nombre de migrants, surtout ceux qui ne parlent ni maltais ni anglais, le téléphone est donc souvent le seul mode de contact possible avec des amis ou membres de la famille. Là aussi, en principe, chaque camp dispose d'un appareil téléphonique à carte que les détenus peuvent librement utiliser. Mais l'application du principe reste soumise à un pouvoir discrétionnaire des surveillants dont la compréhension varie avec la conjoncture et il n'est pas rare que, pour sanctionner un mouvement de rébellion, l'usage du téléphone soit suspendu.

53 En l'absence d'une intervention organisée de travailleurs sociaux qui permettrait aux migrants d'exposer leurs problèmes personnels et de se sentir un peu moins seuls, les seuls soutiens dont ils bénéficient sont le fait d'ONG caritatives (Migrants' Commission of the Catholic Church, Jesuit Refugee Service, Malta Red Cross Society, Islamic Welfare 
Fund), avec les aléas que comporte toute activité bénévole, comme en témoigne le fait que la Croix-Rouge ait dû cesser ses interventions faute de moyens. Et leurs seules distractions régulières sont les moments de prière organisés par ces mêmes ONG. Lorsque le téléphone n'est pas utilisable - parce qu'interdit ou inaccessible - le directeur de la Commission des Emigrants se charge de transmettre les messages des uns et des autres à leurs amis et familles. Mais les détenus n'ont qu'une confiance limitée dans le procédé, notamment parce que les différences linguistiques ne leur permettent pas d'être certains de comprendre les messages ainsi transmis.

$\mathrm{Au}$ final, simplement parce qu'ils sont étrangers et n'ont pas leur place sur le territoire, les migrants sont incarcérés dans des conditions indignes, largement inférieures à celles que connaissent les prisonniers de droit commun détenus à la prison de Corradino $^{32}$. Le gouvernement maltais en convient, mais arguant de ses ressources limitées, «il ne considère pas nécessaire de sacrifier d'autres projets plus prioritaires pour le pays afin de construire un nouveau centre de détention pour immigrants $»^{33}$.

\section{Demandeurs d'asile : l'enfermement comme élément d'un système de dissuasion}

55 Il n'est pas contestable que Malte est matériellement peu équipée pour faire face à des arrivées parfois massives de migrants. Ceci ne saurait cependant suffire à expliquer les choix opérés par le gouvernement pour la gestion de ces arrivées. Il faut, pour les comprendre, tenir compte de la politique d'asile bien particulière de ce pays. A Malte, tout demandeur d'asile arrivé sans titre sur le territoire est d'abord considéré comme un migrant «illégal » et donc placé en détention. Et il reste détenu jusqu'à l'issue de la procédure, qui peut être très longue. C'est ainsi que la plupart des centres de détention sont essentiellement occupés par des demandeurs d'asile en attente de décision. L'examen de la législation dans ce domaine, et de l'application qui en est faite en pratique permet de mesurer à quel point l'enfermement est devenu à Malte un mode de gestion du droit d'asile, malgré les prescriptions du droit international et les protestations de nombreux observateurs, sans toutefois ébranler les positions du gouvernement maltais.

\section{Un droit d'asile en trompe l'oeil}

56 On rappellera que ce que l'on appelle communément le "droit d'asile - et qu'on devrait plutôt nommer le "droit de l'asile» - n'implique aucunement pour les personnes en quête de protection le droit d'obtenir l'asile, mais seulement celui de le demander, les Etats restant maîtres de l'octroyer ou non, en vertu des procédures qu'ils ont mises en place. A cette fin, Malte s'est dotée d'une législation sur l'asile en 2000, le Refugees Act, entrée en application en octobre 2001. La loi prévoit deux types de protection:

57 - le statut de réfugié, au sens de la convention de Genève ${ }^{34}$. Ce statut est matérialisé par la délivrance d'un permis de résidence et, sauf si le réfugié est incarcéré en raison de poursuites ou condamnation judiciaires, un document de voyage lui permettant d'entrer et sortir de Malte sans visa. Son bénéficiaire bénéficie de l'aide sociale et du droit de travailler. 

d'information précisant la confidentialité des renseignements qu'ils donneront, le droit de demander l'assistance d'un avocat et d'entrer en contact avec le représentant du HCR. Cette notice existe au moins en huit langues. Il leur est également fourni un questionnaire (Preliminary Questionnaire), qui une fois rempli, est transmis au Commissariat aux réfugiés. Il ne s'agit toutefois pas d'une formalité obligatoire: le questionnaire est destiné à aider les personnes à formuler leur demande. Vient ensuite 
l'audition par la Commission aux réfugiés : ce sont en général les représentants de la Commission qui se déplacent dans les différents lieux de rétention des étrangers pour recueillir les requêtes et pour procéder aux entretiens ; plus rarement, les demandeurs sont transférés à la Commission pour y être interviewés. Les interviews sont toutes intégralement enregistrées. La présence d'un interprète est presque toujours nécessaire et, pour certaines nationalités il semble parfois difficile d'en trouver, ce qui prolonge la durée de la procédure. L'entretien avec le demandeur n'est pas la seule source d'information du Commissaire : dans certains cas, celui-ci peut laisser du temps au demandeur pour réunir des éléments ou pièces complémentaires nécessaires destinés à étayer sa requête. A l'issue de l'instruction, la Commission donne un avis au gouvernement, qui prend et notifie formellement la décision. De fait, les avis de la Commission sont toujours suivis par le gouvernement. La décision est remise aux demandeurs par les fonctionnaires du camp sous pli fermé.

Selon les observateurs ou avocats rencontrés lors de la mission de la FIDH, les modes d'intervention et d'audition de la Commission sont difficilement critiquables: ni la pression du nombre de demandeurs, ni celle des délais, supérieurs au raisonnable s'agissant de personnes internées, ne semblent altérer l'attention et la rigueur apportées par le Commissaire et ses assistants dans l'examen des demandes d'asile. La plupart s'accordent en revanche pour considérer que les effectifs du Commissariat aux réfugiés sont très insuffisants - ce qu'admet le Commissaire lui-même - pour pouvoir répondre plus vite aux demandes et ainsi éviter l'enfermement ${ }^{37}$. Au-delà de cette question d'effectifs de la Commission, les insuffisances tiennent à l'ineffectivité des moyens d'assistance juridique et d'information et recours prévus par la loi et, surtout, à la durée de la procédure d'appel.

Délais.En principe, selon la loi, le demandeur doit être auditionné dans les huit jours. En réalité, ce délai varie en fonction du taux de remplissage des camps et donc du volume des arrivées. Lors de la mission FIDH (fin février 2004) il était de trois mois; mais à d'autres époques, il a pu atteindre huit à dix mois ${ }^{38}$. Les délais pour avoir communication de la décision prise sont également variables. En principe, les décisions sont communiquées aux demandeurs dans l'ordre chronologique de traitement de la demande. Mais lorsque arrive un bateau transportant un grand nombre de personnes de même nationalité, se trouvant plus ou moins dans la même situation, on attend en général que l'instruction de tous les occupants du bateau soit terminée pour leur notifier en même temps les décisions.

Assistance juridique. Aux termes de la loi, les demandeurs d'asile peuvent, pendant toute la procédure, faire appel à un représentant du HCR et à un avocat. Au stade du recours, ils peuvent bénéficier d'une aide juridictionnelle. En réalité, le HCR n'étant représenté à Malte que par une seule personne, qui a en outre d'autres activités, on voit mal comment elle serait en mesure de répondre à la demande de chacun d'entre eux. L'aide juridictionnelle n'étant prévue qu'au stade du recours, l'accès à un avocat au cours du premier stade de la procédure reste virtuel pour la plupart des demandeurs d'asile qui sont dans l'incapacité d'assumer les frais d'honoraires. Certes, quelques avocats militants se rendent régulièrement dans les différents camps; d'autres, qui ont des clients dans les camps, fournissent à l'occasion de l'information aux autres détenus. Mais cette activité repose sur le bénévolat et à ce titre reste fragile et inorganisée. Est également en cause la formation des avocats: très peu sont compétents dans les matières relatives à l'immigration et à l'asile et la faiblesse économique des clients 
potentiels ne permet guère d'espérer le développement de cette spécialité chez les praticiens. La seule intervention régulière est donc limitée à celle de l'avocate de l'association JRS qui ne peut bien évidemment pas faire face à l'ensemble des demandes des migrants répartis dans les différents centres.

Quant à l'aide juridictionnelle prévue par la loi pour le stade du recours, elle est dans la pratique quasi inexistante. Le système fonctionne très mal, en premier lieu parce qu'il n'est pas expliqué aux demandeurs d'asile internés, aucune information à son sujet n'étant organisée. Pour en bénéficier, il faut en faire la demande par écrit et attendre qu'un avocat soit désigné. Les délais dépassent souvent plusieurs mois et ne semblent pas légalement encadrés. En pratique, il n'est pas rare que cette demande ne soit jamais suivie d'effets, privant les demandeurs d'un droit prévu par la loi maltaise. Lorsqu'un avocat est désigné, son nom n'est pas systématiquement communiqué au demandeur, et rien ne l'oblige à se rendre dans le camp où est interné son client. En effet, au terme de la loi sur l'aide juridictionnelle, c'est au client de se rendre au cabinet de l'avocat, or nous avons vu combien les sorties du camp sont difficiles. L'effectivité du droit à l'aide juridictionnelle repose donc sur la bonne volonté de l'avocat désigné à ce titre ${ }^{39}$.

A ces obstacles s'ajoute le fait qu'aucune visite de travailleur social ou de toute autre personne susceptible d'apporter des renseignements d'ordre pratique ou juridique sur le dispositif applicable en matière d'immigration et d'asile à Malte n'est prévue dans le dispositif de rétention des étrangers et des demandeurs d'asile. Ceux-ci souffrent donc d'un fort déficit d'information concernant les procédures qu'ils pourraient mettre en œuvre ou dans lesquelles ils sont engagés. Ajoutée aux délais très longs qui leur sont imposés à toutes les étapes de la procédure, cette absence d'information contribue à faire régner un sentiment récurrent d'abandon, d'insécurité et de détresse, perceptible lors des entretiens avec les personnes internées.

Recours. En cas de rejet de la demande, est remise en même temps que la décision les concernant une notice (qui existe en plusieurs langues) qui indique aux demandeurs d'asile qu'ils peuvent faire appel dans les deux semaines qui suivent la notification, que leur droit à consulter le HCR et d'avoir recours à un avocat est maintenu, et qu'ils peuvent bénéficier d'une aide juridictionnelle. Le Conseil d'Appel des réfugiés, chargé d'examiner les recours, travaille sur dossier, aucun demandeur n'étant en pratique entendu à ce stade de la procédure. Les décisions de rejet ne sont pas motivées. Elles sont lapidaires, souvent réduites à une ligne hors les formules d'usage ${ }^{40}$. Entre l'entrée en vigueur de la loi de 2000 sur l'asile et le début de l'année 2004, aucun recours présenté devant le Conseil d'Appel des réfugiés contre une décision de rejet d'une demande d'asile n'avait donné lieu à une annulation. Ce caractère systématique, combiné avec le fait qu'aucun demandeur n'est auditionné en appel et avec la non motivation des décisions de rejet des recours, vide la procédure d'appel de toute effectivité en la réduisant à une simple formalité sans incidence sur la décision prise par le Commissaire aux réfugiés.

70 Telle qu'elle est appliquée à Malte, la procédure d'asile fait ainsi fonction de leurre derrière lequel la détention s'impose bien comme système. Celle-ci, on le constate, concerne non seulement des personnes au stade de la première instance et en appel, mais aussi celles dont la demande d'asile a été rejetée dans l'attente de leur éloignement, et parfois des personnes dont la demande d'asile a été acceptée, dans l'attente d'une structure d'hébergement susceptible de les accueillir. Non encadrée par la loi, elle n'est par conséquent pas limitée dans le temps, et se prolonge autant que 
l'exige le traitement de la demande d'asile, voire plus longtemps si le dispositif d'hébergement des réfugiés est insuffisant.

71 Aux insuffisances d'une procédure qui ne respecte pas les droits dus aux demandeurs d'asile, il faut ajouter l'arbitraire et l'incohérence du régime auquel ils sont soumis. Il apparaît en effet que la mise en liberté de certains demandeurs d'asile est décidée de temps à autre par le gouvernement, sans régularité particulière ni critères connus, même si elle concerne plus souvent des personnes internées depuis plus d'un an. Ce fut notamment le cas à la période de noël 2003, où un groupe d'Erythréens a fait l'objet d'une telle mesure de «bienveillance ».

72 A contrario, des personnes ayant obtenu un statut au terme de la procédure restent parfois détenues. Le dispositif d'accueil des réfugiés étant très en deçà des besoins, il est en effet arrivé, notamment au cours du premier semestre 2003, que des personnes qui s'étaient vu reconnaître le droit à un statut de réfugié ou un statut humanitaire soient maintenues dans le camp où elles étaient internées en attendant qu'une solution soit trouvée ${ }^{41}$. A la même époque, ont été maintenus en rétention pour la même raison des Irakiens dont la procédure, en cours, avait été suspendue du fait de la modification du contexte en Irak (intervention américaine en mars 2003) mais à qui, sur recommandation du $\mathrm{HCR}$, il avait été octroyé un droit de séjour temporaire dans l'attente de l'évolution des événements.

Et si le pourcentage de demandeurs qui se voient reconnaître un statut à Malte est relativement élevé au regard des moyennes européennes (de l'ordre de 50\%), il faut savoir que très peu obtiennent le statut de réfugié ( $6,5 \%$ des demandes). A la plupart, seul un droit de séjour "humanitaire » est accordé, qui ne leur ouvre droit qu'à un hébergement en centre ouvert et aux repas quotidiens. Leur liberté de circulation est toute relative, car en pratique les lieux d'hébergement sont très éloignés des centres urbains, mal desservis par de rares moyens de transport qui, de toutes façons, cessent de fonctionner en tout début de soirée, obligeant les personnes soit à rester dans le centre "d'accueil » toute la journée soit, si elles veulent aller en ville, à faire des kilomètres à pied. Il est dans ces conditions extrêmement difficile d'envisager une insertion sociale, qui est de toute façon très limitée pour les bénéficiaires d'un statut humanitaire dans la mesure où ils ne sont pas autorisés à travailler. Compte tenu de cette position de totale dépendance à l'égard des autorités, la «mise en liberté » des bénéficiaires de la protection humanitaire est dans ce contexte très relative. Tout laisse à penser que ce statut s'apparente plus à une sorte de "sauf conduit " destiné à préparer, à sa sortie du camp, le départ de l'étranger qui en est muni vers un autre pays qu'à la reconnaissance d'un droit de rester à Malte. D'une certaine façon, du fait de l'absence de toute politique d'intégration, l'enfermement physique imposé aux demandeurs d'asile pendant la procédure se prolonge en isolement social lorsqu'il a débouché sur un droit de séjour. Les deux régimes s'inscrivant dans la logique de la dissuasion qui caractérise le traitement du droit d'asile à Malte.

\section{En violation des instruments du droit international}

Il faut ici rappeler que l'internement administratif, pratiqué comme mode de gestion des étrangers qui sollicitent une admission au titre de l'asile, est contraire à un certain nombre de prescriptions du droit international. Tout au plus peut-il être admis pour une très courte durée à l'arrivée du demandeur sur le territoire d'un pays, à des fins 
d'identification s'il s'y est présenté sans document de voyage, jusqu'à ce qu'il ait formulé sa demande. Le fait qu'une loi nationale permette la détention des étrangers qui sont entrés ou séjournent irrégulièrement sur le territoire ne suffit pas à justifier son application à l'égard des demandeurs d'asile. C'est ce qui se dégage de l'interprétation qui a été donnée des principaux textes pertinents des Nations Unies et du Conseil de l'Europe en la matière par les instances ad hoc.

Le Comité exécutif du Haut Commissariat des Nations Unies pour les Réfugiés (HCR), s'appuyant sur l'article 14 de la Déclaration universelle des droits de l'Homme selon lequel le droit de chercher asile est un droit de l'Homme, pose comme principe, dans ses Lignes directrices sur les critères applicables à la détention des demandeurs d'asile (1999), que les demandeurs d'asile ne doivent pas être détenus. Seules des exceptions strictement encadrées peuvent être admises. En tout état de cause, il ne suffit pas que la détention appliquée aux demandeurs d'asile soit prévue par la législation nationale des Etats pour être dénuée de caractère arbitraire. Elle doit aussi être conforme à l'article 31 de la Convention de Genève de 1951 relative aux réfugiés, selon lequel les Etats d'accueil ne peuvent appliquer aux réfugiés de sanction du fait de leur entrée ou de leur séjour irrégulier, ni ne doivent appliquer aux déplacements de ces réfugiés d'autres restrictions que celles qui sont «nécessaires " ${ }^{42}$. Dans sa Conclusion $n^{\circ} 44^{43}$, le Comité exécutif du HCR indique que la détention, qui ne doit jamais revêtir un caractère automatique, peut être qualifiée de «restriction nécessaire»: le temps de la détermination de l'identité du demandeur; dans le cadre d'une interview préliminaire, pour identifier le fondement de la demande d'asile (mais en aucun cas pour toute la durée de la procédure de détermination); dans le cas où le demandeur a volontairement cherché à soustraire ses documents de voyage ou d'identité avec l'intention de ne pas coopérer avec les autorités ; pour protéger la sécurité nationale et l'ordre public (ce cas se réfère aux circonstances où le demandeur d'asile a des antécédents criminels ou lorsque, d'évidence, son admission sur le territoire serait susceptible de poser un problème pour la sécurité nationale ou l'ordre public).

Cette conclusion précise que n'entre pas dans le champ des « restrictions nécessaires » une politique de détention visant à dissuader de futurs demandeurs d'asile, ou à inciter des demandeurs d'asile à retirer leur requête. L'invocation d'une urgence, par exemple en cas d'arrivée simultanée d'un très grand nombre de demandeurs d'asile, ne peut non plus justifier leur placement dans des «camps fermés» si ce placement n'est pas accompagné d'une recherche immédiate de solutions.

La Sous-Commission de la promotion et de la protection des droits de l'Homme des Nations Unies,dans sa Résolution 2000/21 sur la détention des demandeurs d'asile (2000), met en garde contre certaines pratiques et politiques de détention susceptibles de contrevenir aux principes, normes et règles internationaux relatifs aux droits de l'Homme ou de dissuader des personnes de chercher refuge contre la persécution, « prie instamment les Etats de respecter leurs obligations internationales respectives s'agissant des pratiques et politiques de détention à l'égard des demandeurs d'asile » et les« encourage à adopter d'autres modalités que la détention $»^{44}$.

78 Le Groupe de travail des Nations Unies sur la détention arbitraire estime pour sa part que "tout demandeur d'asile ou immigrant mis en rétention doit être présenté à bref délai à une autorité judiciaire ou autre ", et qu' "un délai maximum doit être prévu par la loi, la rétention ne pouvant en aucun cas être illimitée ou d'une durée excessive $»^{45}$. 
79 Le Comité des ministres du Conseil de l'Europe, dont Malte est un des membres, se réfère, dans sa Recommandation sur les mesures de détention des demandeurs d'asile, à l'article 5 de la Convention européenne des droits de l'Homme et des libertés fondamentales de 1950 pour formuler un certain nombres de principes en vue d'encadrer strictement les conditions auxquelles un demandeur d'asile peut être placé en détention. Ils impliquent, lorsqu'une personne est régulièrement arrêtée ou détenue " pour l'empêcher de pénétrer irrégulièrement dans le territoire, ou contre laquelle une procédure d'expulsion ou d'extradition est en cours", que cette personne soit "informée, dans le plus court délai et dans une langue qu'elle comprend, des raisons de son arrestation", et qu'elle ait "le droit d'introduire un recours devant un tribunal, afin qu'il statue à bref délai sur la légalité de sa détention et ordonne sa libération si la détention est illégale ${ }^{46}$.

80 L'Assemblée parlementaire du Conseil de l'Europe invite, dans sa Recommandation 1327 relative à la protection et au renforcement des droits de l'Homme des réfugiés et des demandeurs d'asile en Europe les Etats membres à recourir en priorité à des méthodes non privatives de liberté, comme les systèmes de surveillance, l'obligation de se présenter régulièrement devant une autorité, la liberté sous caution ou d'autres systèmes de garantie ; à élaborer et diffuser des critères précis pour déterminer quels demandeurs d'asile doivent être détenus; et à introduire dans leur législation sur l'asile des règles fixant la durée maximale de détention des demandeurs d'asile.

81 Enfin, s'agissant des mineurs, la plupart des textes mentionnés excluent a priori la détention des demandeurs d'asile mineurs d'âge, notamment lorsqu'ils sont nonaccompagnés. La Convention des Nations Unies relative aux droits de l'enfant de 1990, qui invite les Etats signataires à un traitement particulier des enfants demandeurs d'asile ou réfugiés pour le respect des principes qu'elle énonce, leur demande dans l'article 37.b de veiller à ce que «nul enfant ne soit privé de liberté de façon illégale ou arbitraire ». Elle précise que «l'arrestation, la détention ou l'emprisonnement d'un enfant doit être en conformité avec la loi, n'être qu'une mesure en dernier ressort, et être d'une durée aussi brève que possible ».

82 Au regard des normes fixées par le droit international, il est clair que la pratique maltaise constitue une violation des principes qui encadrent la détention des demandeurs d'asile, ce dont s'inquiètent régulièrement les ONG et les instances des Nations Unies et du Conseil de l'Europe qui se sont exprimées sur la situation des demandeurs d'asile à Malte. C'est ainsi que le HCR a fait part à plusieurs reprises aux autorités maltaises de ses préoccupations quant à la détention prolongée imposée aux demandeurs d'asile. En novembre 2003, la porte-parole de sa délégation à Rome rappelait à propos de la situation maltaise qu' « en principe, les demandeurs d'asile n'ont pas à être détenus, sauf à titre tout à fait exceptionnel, par exemple lorsqu'il est nécessaire de procéder à la vérification de leurs documents. Même dans ce cas, limité aux situations où les demandeurs d'asile sont porteurs de faux documents ou n'en ont pas du tout, la détention n'est opportune que si le refus de coopérer est manifeste. Pour ce qui concerne l'instruction de la demande d'asile, la détention ne peut dépasser le stade de l'interview préliminaire, et en aucun cas durer jusqu'à la décision. La détention d'un demandeur d'asile n'est justifiée que s'il s'agit d'un criminel ou quand sont en jeu la sécurité nationale et l'ordre public ». Elle a également souligné que "les sanctions prévues contre les migrants qui franchissent illégalement les frontières ne peuvent être appliquées aux demandeurs d'asile, dans la mesure où ceux-ci peuvent ne pas avoir été capables de répondre aux formalités légales d'entrée ${ }^{47}$. 

très préoccupée par la politique de détention illimitée à l'égard des demandeurs d'asile. L'association relève que cette détention peut atteindre une à deux années et concerne toutes les catégories de demandeurs d'asile y compris les plus vulnérables. Elle réitère sa demande aux autorités maltaises de prendre des mesures afin que, en conformité avec les standards internationaux, les demandeurs d'asile ne soient détenus que lorsqu'à un niveau individuel des motifs légitimes justifient un tel traitement, à condition qu'il n'y ait pas d'alternative à cette détention, et seulement pour une courte durée $e^{48}$.

Dans le rapport qu'il a rendu public en janvier 2004 sur la visite qu'il a effectuée à Malte trois mois auparavant, Alvaro Gil-Robles, Commissaire aux droits de l'Homme du Conseil de l'Europe, réaffirme que «par principe [la place des demandeurs d'asile] n'est pas en détention lorsqu'ils n'ont pas commis de délit pénal, et que les Etats doivent tout mettre en cuvre afin d'éviter l'incarcération $»^{49}$.

C'est sans conteste l'association JRS Malta qui a fourni l'argumentaire le plus élaboré pour contester avec fermeté la pratique de la détention systématique des demandeurs d'asile, à laquelle elle a consacré plusieurs publications. Adossant sa position à l'analyse des textes nationaux et internationaux pertinents en matière de respect des droits de l'Homme et des droits des réfugiés, JRS plaide pour la mise en liberté des demandeurs d'asile internés à Malte, et énonce plusieurs recommandations précises associées à cette position ${ }^{50}$.

\section{Résistance maltaise et complaisance européenne}

Ces mises en garde et recommandations restent apparemment sans écho. On constate en effet que les autorités maltaises n'ont jamais remis en cause le recours à la détention, qui, on l'a vu, relève plus d'une politique de dissuasion que du registre de la réponse conjoncturelle à une situation de crise. Il faut dire qu'elles sont en cela sinon soutenues, du moins partiellement légitimées par les instances de l'Union européenne.

Le gouvernement maltais a eu à plusieurs reprises l'occasion de s'exprimer, en la personne du ministre de l'Intérieur, sur sa politique d'enfermement. Outre le fait qu'il la justifie au nom des spécificités du pays (petite taille, position géographique), du contexte migratoire (augmentation spectaculaire du nombre d'arrivées au cours de cinq dernières années) et de la protection de l'ordre public, il ne fait jamais de distinction entre la situation particulière des demandeurs d'asile et celle des autres étrangers. Au contraire, la confusion est régulièrement entretenue, dans les déclarations officielles, entre ces deux catégories, par l'utilisation générique, pour les désigner, de l'expression « immigrants illégaux » (« illegal immigrants ») même lorsqu'il est question des procédures d'asile ou de la reconnaissance du statut de réfugié les concernant. Elle se vérifie encore dans l'affirmation du ministre de l'Intérieur, rapportée par la presse, selon laquelle - contre toute évidence - la loi sur l'immigration interdirait de laisser les demandeurs d'asile en liberté ( $L^{\prime}$ 'Immigration Act a rendu la libération des demandeurs d'asile impossible $»^{51}$ ).

Si l'accélération des procédures d'asile semble être un des objectifs des autorités maltaises, ce dont pourrait témoigner un projet de loi déposé en janvier 2004 relatif à l'augmentation des effectifs des instances chargées de la détermination du statut de réfugié, aucun signe ne permet aujourd'hui de penser qu'elles souhaitent mettre fin au 
système de détention des demandeurs d'asile, sauf, éventuellement, au cas où la détention se prolongerait au-delà du raisonnable («le gouvernement prévoit de(...) libérer les immigrants irréguliers dont la détention dépasse une durée raisonnable ${ }^{52}$ ).

Cette position n'a pas été ébranlée par le rapport de visite du Commissaire aux droits de l'Homme du Conseil de l'Europe. Aux observations de ce dernier sur ce point, il est répondu: "Le gouvernement maltais apprécie les commentaires du Commissaire, mais ne considère pas qu'il est dans l'intérêt de Malte de changer sa politique de détention. Si les 2000 personnes entrées illégalement à Malte au cours des deux dernières années avaient été immédiatement relâchées, elles auraient causé des perturbations sur le marché du travail et des problèmes importants quant à leur hébergement $»^{53}$. Et si au mois de février 2004, le ministre de l'Intérieur déclarait qu'un projet de loi serait bientôt présenté au Parlement, prévoyant la possibilité de contester en justice les cas de durée excessive de rétention, il ajoutait que le principe même de la rétention ne pouvait être remis en cause, car il serait contraire à l'intérêt de la nation de laisser les immigrants illégaux en liberté (« Le ministre Borg a déclaré qu'il était contre l'intérêt national d'avoir des immigrants irrégulier libres $\left.\|^{54}\right)$.

De leur côté les instances de l'Union européenne, qui ont eu à se prononcer sur la question de l'internement des demandeurs d'asile à l'occasion de l'adhésion de Malte dans l'Union, se sont montrées très compréhensives dans leur analyse de la situation, en mettant en avant les difficultés inhérentes à la taille du pays et la longueur des procédures d'asile. Ainsi pour le Conseil européen interpellé le 18 septembre 2003 par une parlementaire européenne sur les dispositions qu'il entendait prendre pour s'assurer que, à quelques mois de l'adhésion de Malte à l'Union européenne, ce pays se conforme à ses obligations internationales et à l'acquis de l'Union dans le domaine de l'accueil des demandeurs d'asile, il se déclarait conscient de la situation mais aussi du fait que "Malte dispose d'une capacité d'accueil limitée des immigrés clandestins et des

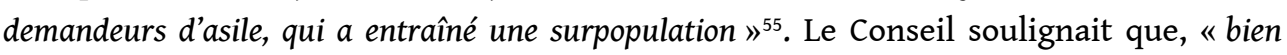
que n'ayant pas encore trouvé de solution adéquate, les autorités maltaises s'efforcent de garantir pour les immigrés clandestins et les demandeurs d'asile des conditions d'accueil aussi correctes que les circonstances le permettent, ne serait-ce que compte tenu de leur nombre ${ }^{56}$. Dans ce contexte, il estimait "nécessaire d'évaluer les conditions de détention des demandeurs d'asile en liaison étroite avec la capacité d'accueil réelle de Malte, qui est limitée ${ }^{57}$. On rencontre la même complaisance à la Commission européenne. Chargée de suivre les progrès des Etats candidats à l'adhésion à l'Union européenne au regard des normes communautaires, elle a rendu, depuis 1999, plusieurs rapports sur chacun d'entre eux. Dans son rapport de novembre 2003, elle « félicite Malte pour les progrès accomplis en 2003 [mais] encourage le pays à faire des efforts en ce qui concerne (...) le droit d'asile ». Elle estime qu'« en particulier, Malte devrait réduire la durée de ses procédures d'asile et améliorer les capacités d'accueil et de détention des demandeurs». En mars 2004, elle observe que " pour ce qui est de la mise en cuvre de la législation sur l'asile, Malte doit consentir encore des efforts pour la procédure accélérée, l'assistance juridique et l'intégration des personnes ayant obtenu le statut de demandeurs d'asile. Compte tenu de la longueur de la procédure, les demandeurs d'asile sont maintenus dans des centres de détention pendant plusieurs mois $»^{58}$.

\section{Quelques propositions pour éviter le syndrome maltais}

91 Le droit d'asile ne se résume pas au principe de non refoulement. En se contentant de ne pas renvoyer les personnes à qui elles reconnaissent le droit à une protection sans 
leur assurer les garanties d'une intégration durable, et en fermant les yeux sur les conditions de leur départ de Malte vers d'autres pays d'Europe, les autorités maltaises n'apportent qu'une réponse partielle aux problèmes qui leur sont posés. Au-delà, l'attitude de l'Union européenne telle qu'elle est exprimée par la voix de la Commission et du Conseil, à laquelle fait écho le silence significatif des partenaires de Malte dans l'UE, est un élément déterminant pour l'analyse de la politique maltaise de détention des migrants et des demandeurs d'asile. Tout se passe comme s'il suffisait que ce pays soit doté d'une législation conforme aux normes internationales en matière d'accès au territoire et d'asile pour que les apparences soient sauves. La donnée européenne est par conséquent indissociable de la recherche de solutions susceptibles de mettre fin au régime d'internement systématique pratiqué à Malte, et d'éviter que le "syndrome maltais " se généralise. C'est pourquoi, en conclusion de son rapport de mission, la FIDH formule une double série de recommandations.

La première s'adresse aux autorités maltaises : les arguments invoqués pour justifier le dispositif d'exception appliqué aux migrants et aux demandeurs d'asile, en se référant aux caractéristiques géopolitiques et topographiques du pays, ne résistent pas à la confrontation avec les prescriptions des textes qui engagent Malte au plan international et régional, et avec l'interprétation qu'en font les organes chargés de veiller à leur application. Mais il apparaît que c'est moins la législation de ce pays que ses pratiques qui doivent et peuvent être modifiées afin de mettre les modalités d'accueil des migrants en situation irrégulière, comme des demandeurs d'asile et des réfugiés, en conformité avec les principes et les obligations auxquels Malte a souscrit au travers des différentes conventions internationales et régionales qu'elle a ratifiées.

Cependant, il faut être conscient de ce que les spécificités de Malte ne seront pas réglées par des réformes d'ordre uniquement national. $\mathrm{Du}$ fait de sa position géographique, le pays est et restera un point de passage dans les parcours des migrants et des personnes en quête de protection en provenance d'Afrique et du Moyen-Orient vers l'Europe. L'entrée de Malte dans l'UE ne peut que renforcer son statut de terre de transit et laisse même prévoir une augmentation des demandes d'asile qu'elle aura à traiter. La seconde série de recommandations vise par conséquent les instances européennes. Elle leur rappelle que l'application mécanique du règlement Dublin II auquel Malte, comme ses partenaires de l'Union, est désormais soumise, va l'obliger à réadmettre les requérants d'asile qui seront passés par son sol avant de se rendre ailleurs dans un autre pays de l'Union. Il risque de s'ensuivre un grave déséquilibre, préjudiciable à la qualité de l'accueil des demandeurs d'asile et du traitement de leur dossier, et par ricochet de susciter, à l'égard d'éventuels candidats à l'asile, une politique de refoulement accru de la part des autorités maltaises, au détriment des principes de protection.

Deux solutions, non nécessairement exclusives l'une de l'autre, sont envisageables : la première consisterait en une dérogation à l'application du règlement Dublin II, permettant à Malte d'être déchargée de la responsabilité du traitement d'une demande d'asile émanant d'un étranger qui aurait transité par son territoire avant d'aller ailleurs en Europe ${ }^{59}$. L'autre solution, raisonnable au vu des contraintes économiques et sociales que représente l'intégration de nouvelles populations dans un aussi petit pays, serait de permettre aux personnes reconnues réfugiées ou s'étant vues reconnaître un statut humanitaire à Malte de s'établir librement et légalement dans un autre Etat membre. 

depuis son adhésion, ne peuvent rester silencieux devant l'inacceptable régime d'enfermement que ce pays maintient à l'égard des migrants. Car au-delà de Malte, c'est le rapport de la plupart des pays nouvellement adhérents à la gestion des flux migratoires qui doit être posé. Si l'on peut espérer que les différences de traitement constatées dans les procédures mises en œuvre en matière d'asile seront quelque peu gommées par l'application des directives existantes ou à venir - et à la condition que la latitude laissée aux uns et aux autres par des clauses facultatives ne légitime pas un droit d'asile à géométrie variable - on ne peut négliger le fait que ces « nouveaux » vont pour la plupart devenir les premiers pays d'accueil des migrants en route vers l'UE.

Sauf à mettre en œuvre au plus vite des solutions propres à corriger les déséquilibres qui ne peuvent qu'en résulter, l'Union européenne prend le risque de favoriser, à l'instar de ce qui se passe à Malte, le développement d'une gestion avant tout sécuritaire des flux migratoires et, comme c'est le cas dans d'autres régions du monde, notamment en Australie, une politique de rejet des demandeurs d'asile par leur mise à l'écart physique ${ }^{60}$.

\section{NOTES}

*. Catherine Teule, secrétaire générale de la Ligue française des droits de l'Homme, et Claire Rodier, membre du GISTI (groupe d'information et de soutien des immigrés) ont effectué, pour la Fédération Internationale des Droits de l'Homme (FIDH), une mission sur les conditions de détention des migrants et des demandeurs d'asile à Malte au mois de février 2004. Cet article est largement tiré du rapport de mission : "Enfermer les étrangers, dissuader les réfugiés : le contrôle des flux migratoires à Malte »; FIDH, rapport $\mathrm{n}^{\circ}$ 403, septembre 2004. Ce document est disponible sur :http://www.fidh.org/IMG/pdf/mt403f.pdf

1. Coordination française pour le droit d'asile, mars 2002.

2. Le règlement « Dublin » $n^{\circ}$ 343/2003 du 18 février 2003 implique que le pays qui, en le laissant pénétrer illégalement sur son sol, a fait entrer un étranger dans l'Union européenne, est « responsable» du traitement de la demande d'asile de cet étranger, même si elle est déposée bien plus tard dans un autre Etat de l'Union.

3. Le règlement Eurodac, qui prévoit le relevé des empreintes dactylocopiques des demandeurs d'asile et de toute personne qui franchit illégalement une des frontières de l'UE, est destiné à faciliter l'application du règlement Dublin.

4. UNHCR: Asylum levels and trends: Europe and non-European industrialized countries, 2003, Genève, février 2004. Selon le rapport du HCR, le nombre de demandes d'asile enregistrées dans l'UE à 15 s'est élevé à 288400 en 2003, en décroissance de $22 \%$ par rapport à l'année précédente. Dans le même temps, les demandes d'asile enregistrées dans les 10 nouveaux pays membres avaient augmenté de 16\%, pour s'élever à 37 300, soit 11\% du total de l'UE à 25.

5. La notion de "pays sûr », discutée à l'occasion de l'élaboration d'une directive communautaire sur la procédure applicable aux personnes se réclamant de la qualité de réfugié, consiste à considérer que certains pays, dont la liste pourrait être préétablie et s'imposerait à tous les Etats membres de l'UE, ne sont pas susceptibles, au regard du respect des principes démocratiques et 
relatifs aux droits de l'Homme, de "générer " des réfugiés. La notion de "pays-tiers sûr ", également discutée, permettrait de rejeter la demande d'asile présentée par un étranger au motif qu'il aurait pu trouver protection dans un pays par lequel il a transité avant d'arriver en Europe.

6. Le concept d'« asile interne» permet de rejeter la demande d'asile d'une personne menacée dans une région de son pays mais qui aurait pu trouver asile dans une autre région, au lieu de le demander à l'étranger.

7. Sauf précision contraire, toutes les observations mentionnées dans cet article sur la situation à Malte ont été recueillies directement par les auteurs, membres de la mission FIDH.

8. Ces caractéristiques ont longtemps servi d'argument aux opposants à l'adhésion : les Maltais craignaient de se diluer dans la grande Europe et de perdre leurs spécificités culturelles et sociales nationales très marquées par l'insularité.

9. En 2002, par exemple, sur un total de 1686 arrivées par voie de mer, 466 personnes avaient été recueillies lors d'opérations assurées par l'armée. En 2003, sur un total de 497 personnes débarquées, elles étaient 80 . On peut noter que certaines des embarcations transportant ces migrants - bateaux de pêche généralement - contenaient plus de 200 passagers.

10. Refugees Actdu 1er octobre 2001, " To make provisions relating to and establishing procedures with regard to refugees and asylum seekers ».

11. Tonio Borg, ministre de l'Intérieur, à la «Conference on human rigths and the treatment of asylum seekers ", Malta dive-news, 20 novembre 2003.

12. Déclaration de Tonio Borg, ministre de l'Intérieur, à l'occasion de la venue à Malte, le 27 juillet 2003, du ministre de l'Intérieur italien.

13. Conseil de l'Europe, Commentaires et observations des autorités maltaises concernant le rapport du Commissaire aux droits de l'Homme sur sa visite à Malte, « Rapport de M. Alvaro GilRoblès, Commissaire aux droits de l'Homme, sur sa visite à Malte", Strasbourg, p. 14. www.coe.int/T/E/Commissioner_H.R/Communication_Unit/Documents/ pdf.CommDH(2004) $4 \mathrm{Fpdf}$

14. "Le pays doit (...) étendre sa législation aux procédures de détention des personnes ayant fait l'objet d'arrêtés d'expulsion ou qui se sont vues refuser l'entrée sur le territoire " in Commission européenne, Rapport global de suivi des préparatifs menés par Malte en vue de son adhésion, Rapport de la Commission européenne au Conseil en vue de l'adhésion de Malte du 5 novembre 2003. http:// europa.eu.int/comm/enlargement/report_2003/pdf/cmr_mt_final_fr.pdf.

15. Sont traditionnellement considérées comme "vulnérables»: personnes âgées, femmes allaitant des nouveaux-nés, enfants non sevrés, mineurs non-accompagnés, malades, femmes dans les dernières semaines de leur grossesse.

16. Egypte, Maroc, Tunisie, Algérie. Malte a, par ailleurs, signé en 2003 des accords de coopération avec 11 pays : Albanie, Algérie, Chypre, Egypte, Espagne, France, Grèce, Israël, Italie, Libye, Tunisie, Turquie.

17. Voir la note 2 .

18. Comité européen pour la prévention de la torture. Voir : http://cpt.coe.int/fr/

19. «Report to the Maltese Government on the visit to Malta carried out by the European Committee for the Prevention of Torture and Inhuman or Degrading Treatment or Punishment (CPT) from 13 to 18 May $2001 »$, Strasbourg, juillet 2003. Voir le site suivant : http:// www.cpt.coe.int/documents/mlt/2002-16-inf-eng.htm

20. . Voir notamment Bernardot M., « Existe-il un modèle français du camp ? », conférence du 25 janvier 2002. Il rappelle la définition qu'en donne R. Barthes: le camp est un «regroupement imposé et arbitraire de civils en dehors du système pénitentiaire pour une durée indéterminée, visant à les enfermer, les rééduquer ou les faire travailler ». Voir aussi les publications de Migreurop : http:// www.migreurop.org/

21. . Par exemple, selon le ministre de l'Intérieur, en décembre 2002 le nombre d'étrangers détenus s'élevait à 900, en octobre 2003 à 550 et à 240 en février 2004. Ces chiffres peuvent être 
rapprochés de ceux des « entrées » illégales : 1 686, en 2002 (700 personnes reconduites la même année) ; 497 en 2003 (300 reconduites). Encore faut-il préciser que ces données ne concernent que les arrivées par voie maritime et non celles enregistrées à l'aéroport, ni les interpellations sur le territoire. On comprend qu'appliquées à un nombre d'entrées représentant 5\%o de la population (soit la moitié des naissances annuelles), comme ce fut le cas sur la période 2002-2003, et pour une longue durée, les structures de détention se révèlent absolument inadaptées.

22. . " Follow-up response of the Maltese Government to the report on the visit to Malta carried out by the CPT from 13 to 18 May $2001 »$. Traduction libre. Disponible sur: http:// www.cpt.coe.int/documents/mlt/2003-34-inf-eng.htm

23. Conseil de l'Europe, Rapport de M. Alvaro Gil-Roblès, op. cit, p. 7.

24. Un groupe d'Erythréens a été libéré en décembre 2003, après une campagne d'Amnesty International dénonçant le renvoi de leurs compatriotes un an plus tôt.

25. Annual report of the Ombudsman, "The detention of illegal immigrants by the Police - an own motion investigation by the Ombudsman", 24 mai 2002, pp. 105-106. Voir: http://www.euroombudsman.eu.int/report02/pdf/en/rap02en.pdf

26. Conseil de l'Europe, Rapport de M. Alvaro Gil-Roblès, Commissaire aux droits de l'Homme, sur sa visite à Malte, 12 février 2004, p. 7.

27. A Ta'Kandja, les gardiens changeant trop souvent, ils ne peuvent assurer cette distribution et ce sont les détenus qui se débrouillent.

28. Le JRS est la seule ONG qui soit vraiment présente dans les centres de détention des étrangers. Il est l'auteur de plusieurs rapports très sévères sur les conditions de détention des étrangers (notamment : "Detention of asylum seekers in Malta : a human rights perspective ", juillet 2003).

29. Dudley M., «Two Australian national policies on self-injury and suicide : a submission on behalf of Suicide Prevention to the Human Rights and Equal Opportunity Commission enquiry concerning children in detention ", mai 2002.

30. Conseil de l'Europe, Rapport de M. Alvaro Gil-Roblès, Commissaire aux droits de l'Homme, sur sa visite à Malte, 12 février 2004, p. 15.

31. Grech Marguerat P., propos rapportés par The Times of Malta, 4 novembre 2003. Traduction libre.

32. Cette prison a été récemment rénovée et les travaux réalisés se sont élevés à 5,5 millions de

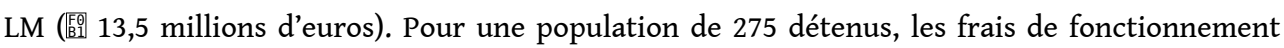
sont évalués à 2 millions de LM (苞 4,9 millions d'euros). Chiffres cités par Tonio Borg, ministre de l'Intérieur, Malta di-ve-news, 20 novembre 2003.

33. Conseil de l'Europe, Rapport de M. Alvaro Gil-Roblès, op. cit., p. 15.

34. Convention de Genève, art. 1 : (toute personne) « craignant avec raison d'être persécutée du fait de sa race, de sa religion, de sa nationalité, de son appartenance à un certain groupe social ou de ses opinions politiques ".

35. Refugee Act, art. 8(7). Traduction libre.

36. Sur ces notions, voir la note 5 .

37. Dans son rapport de visite, le Commissaire aux droits de l'Homme du Conseil de l'Europe, Alvaro Gil-Roblès, estime nécessaire "que les autorités maltaises fournissent aux organes compétents en matière d'asile - Commissaire aux réfugiés et Conseil d'Appel des réfugiés - un personnel permanent plus important afin de leur permettre de prendre des décisions plus rapidement tout en continuant à accorder le temps nécessaire à l'écoute et à l'analyse des dossiers ». Conseil de l'Europe, Rapport de M. Alvaro GilRoblès, op. cit., p. 8. C'est probablement en réponse à cette recommandation qu'un projet de loi présenté par le gouvernement en janvier 2004 donne la possibilité au Premier ministre de nommer des Commissaires assistants et de créer au sein du Conseil d'Appel des réfugiés plusieurs chambres pour l'examen des recours.

38. Estimation JRS, Detention of asylum seekers in Malta: a human rights perspective, juillet 2003. 
39. L'association JRS rapporte le cas de vingt-cinq demandeurs d'asile qui, plus de quatre mois après avoir, avec difficultés, formulé une demande d'aide juridictionnelle, n'avaient toujours pas pu entrer en contact avec les avocats qui avaient été désignés pour les assister, ceux-ci ne se considérant pas pour leur part saisis par leurs clients.

40. Dans son rapport de visite, Alvaro Gil-Roblès se dit «surpris de constater que la plupart de ces 'décisions' consistaient en une simple lettre ne comprenant aucun exposé des motifs et se limitant parfois à une seule phrase ", Conseil de l'Europe, Rapport de M. Alvaro Gil-Roblès, point 23, op. cit., p. 8.

41. JRS mentionne qu'à cette période jusqu'à 265 personnes ont été ainsi maintenues en détention, sans savoir que le Commissaire aux Réfugiés leur avait octroyé un statut, jusqu'à ce que des places se libèrent dans un centre d'accueil. C'est finalement la décision du gouvernement de transformer certains bâtiments jusqu'alors utilisés comme camps de détention en centres ouverts qui a permis leur mise en liberté.

42. Pendant longtemps, Malte a maintenu une réserve concernant l'application de l'article 31 de la Convention de Genève, au terme de laquelle cet article s'appliquerait dans la mesure de sa compatibilité avec les problèmes spécifiques du pays. Mais cette réserve a été levée au mois de févier 2002, sans que la pratique change.

43. Conclusion $n^{\circ} 44$ (XXXVII) EXCOM du HCR relative à la détention des demandeurs d'asile et des réfugiés (1986).

44. Sous Commission de la promotion et de la protection des droits de l'Homme des Nations Unies, Résolution 2000/21 sur la détention des demandeurs d'asile, 2000.

45. Commission des droits de l'Homme des Nations Unies, Rapport du groupe de travail sur la détention arbitraire, annexe IIb,délibération $n^{\circ} 5$ sur la situation des immigrants et des demandeurs d'asile, 28 décembre 1999, p. 29.

46. Conseil de l'Europe, Rec (2003)5,16 avril 2003, disponible sur le lien suivant: https:// wcm.coe.int/ViewDoc.jsp?id=2107\&Lang=fr

47. Traduction libre (de l'anglais) des propos de Mme Boldrini, porte-parole du HCR à Rome reproduits dans une dépêche de l'Independent (Malte), 16 novembre 2003.

48. Amnesty International, Concerns in Europe and Central Asia june-december 2003, Malta, avril 2004.

49. Conseil de l'Europe, Rapport de M. Alvaro Gil-Roblès, op. cit., p. 5.

50. Voir notamment JRS Malta, Detention of asylum seekers in Malta : a human rights perspective, policy recommendations, juillet 2003.

51. Times of Malta, 10 mai 2003. Traduction libre.

52. Times of Malta, 21 novembre 2003. Traduction libre.

53. Conseil de l'Europe, Rapport de M. Alvaro Gil-Roblès, op. cit., p. 14.

54. MaltaMedia.com, 9 février 2004. Traduction libre.

55. Question écrite P-2875/03 posée par Hélène Flautre (Verts/ALE) au Conseil (18 septembre 2003), réponse du 4 avril 2003.

56. Ibid.

57. Ibid.

58. Rapports de la Commission européenne au Conseil en vue de l'adhésion de Malte du 5 novembre 2003, http://europa.eu.int/comm/enlargement/report_2003/pdf/ cmr_mt_final_fr.pdf. et du 24 mars 2004, http://europa.eu.int/scadplus/leg/fr/lvb/e06112.htm.

59. Voir, sur ce point, la Recommandation 1327 de l'Assemblée parlementaire du Conseil de l'Europe (1997) qui invite (pt 8-vi) les Etats membres à modifier [le mécanisme « Dublin »] «afin de permettre aux demandeurs d'asile d'exprimer un choix quant au pays où ils souhaitent demander asile, dès lors qu'ils peuvent faire la preuve d'un lien avec ce pays ». Voir: http://assembly.coe.int/ Documents/AdoptedText/TA97/FREC1327.HTM

60. La Pacific solution, pratiquée par le gouvernement australien, consiste à placer les demandeurs d'asile dans des camps de détention parfois situés sur le territoire des micro-Etats qui entourent l'Australie. Un rapport du groupe de travail sur la détention arbitraire de la Commission des 
droits de l'Homme des Nations Unies dénonce le traitement des demandeurs d'asile dans les camps australiens, 24 octobre 2002.

\section{RÉSUMÉS}

La République de Malte, située à mi-chemin entre le continent européen et l'Afrique, est depuis toujours à la croisée des circuits migratoires. Avec l'élargissement du $1^{\text {er }}$ mai 2004, Malte est devenue une des portes d'entrée de l'Union européenne pour des centaines de personnes qui échouent par choix ou plus souvent par hasard sur ses plages. Pour faire face à ce phénomène, les autorités maltaises ont mis en œuvre une politique d'enfermement systématique des étrangers qui arrivent irrégulièrement, y compris lorsqu'il s'agit de demandeurs d'asile. Les conditions matérielles de détention ne répondent pas aux standards minimums en matière d'hygiène et de respect de la vie privée. Ceux qui sollicitent l'asile sont soumis à un régime incompatible avec les normes internationales. Par ailleurs, même si Malte accorde un certain nombre de permis de séjour à caractère humanitaire, la sortie des « camps » ne se traduit pas par une intégration dans le pays, où aucune politique d'accueil des réfugiés n'est organisée. La plupart d'entre eux rejoignent donc d'autres pays de l'UE où ils ont vocation à devenir sans-papiers. Mais c'est bien de l'Union européenne que dépendent les solutions pour éviter que Malte ne transforme sa politique de contrôle des frontières en machine répressive aux seules fins de dissuader les réfugiés.

Situated half way between Africa and Europe, the Republic of Malta has always been at the crossroads of migration routes. Since the May $1^{\text {st }} 2004$ European enlargement, Malta has become one of the entries to the European Union for hundreds of people who choose or happen to end up on its beaches. To face this phenomenon, the Maltese authorities have started a policy of systematic detention of all illegal immigrants arriving, whether they seek asylum or not. The material conditions of detention do not respect the minimum hygiene and private life standards. Those detained seeking asylum are faced to a regime incompatible with international norms. Moreover, even if Malta gives a statistically large number of humanitarian permits, getting out of the «camps » does not mean integrating the Maltese society, in which there is no organised integration policy for refugees. Most of them therefore seek to join other European countries where they end up being clandestine. But the solutions to avoiding Malta from transforming its policies of frontiers' control into a repressive machine aiming at only dissuading refugees from coming can only come from the European Union.

\section{INDEX}

Mots-clés : Camps, Malte, demandeurs d'asile, réfugiés, Union européenne 


\section{AUTEURS}

\section{CLAIRE RODIER}

Claire RODIER est membre du GISTI (groupe d'information et de soutien des immigrés). Elle a effectué avec Catherine Teule, pour la Fédération Internationale des Droits de l'Homme (FIDH), une mission sur les conditions de détention des migrants et des demandeurs d'asile à Malte en février 2004.

\section{CATHERINE TEULE}

Catherine TEULE est secrétaire générale de la Ligue française des droits de l'Homme. Elle a affectué avec Claire Rodier une mission pour la FIDH sur les conditions de détention des migrants et des demandeurs d'asile à Malte en février 2004. 